\title{
HOMOGENEOUS CONES AND ABELIAN THEOREMS
}

\author{
TATJANA OSTROGORSKI
}

\author{
Département de Mathématiques \\ Université de Montpellier III, France \\ Mathematical Institute \\ Belgrade, Yugoslavia
}

(Received October 31, 1996 and in revised form September 26, 1997)

\begin{abstract}
This paper deals with analysis on homogeneous cones in $\mathbb{R}^{\boldsymbol{n}}$. This subject has its origins in one-dimensional topics that are connected, often implicitely, with some group properties. The homogeneous cones are open convex cones in $\mathbb{R}^{\boldsymbol{n}}$ that are at the same time homogeneous spaces, and they are more general than the classical, or symmetric cones. As an example of application of the theory of homogeneous cones we study the asymptotic behaviour of some integral transforms.
\end{abstract}

KEY WORDS AND PHRASES: Homogeneous cones, Vinberg algebras, Integral transforms, Abelian Type Theorems, Laplace and Stieltjes transforms, Riemann-Liouville operator.

1991 AMS SUBJECT CLASSIFICATION CODES: 43 A 85, 44 A 10, 17 D 99.

\section{INTRODUCTION}

The purpose of this paper is to find an $n$-dimensional analogue of the classical Abelian Theorem for the Laplace Transform (see Widder [1])

$$
\text { If } f(t) \sim t^{\alpha}, t \rightarrow \infty, \quad \text { then } \mathscr{L} f(t) \sim \Gamma(\alpha+1) t^{\alpha}, t \rightarrow \infty .
$$

(Here $f$ is a positive function defined on $\mathbb{R}_{+}=(0, \infty)$, the parameter $\alpha>0$ and the Laplace transform is taken in the form $\mathscr{L} f(t)=\int_{0}^{\infty} \mathrm{e}^{-u / t} f(u) d u / u$.)

This theorem relies mainly on the group properties of $\left(\mathbf{R}_{+},,^{\circ}\right)$ (the multiplicative group of positive reals). Indeed, the functions $t \mapsto t^{\alpha}$ are the automorphisms of this group, $d u / u$ is the invariant measure and the kernel $e^{-u / t}$ is a homogeneous function (of two variables).

As $n$-dimensional counterparts of the half-line $\mathbb{R}_{+}$we shall take homogeneous cones. These are cones in $\mathbb{R}^{n}$ endowed with a group operation (see Definition 2.1 below). The theory of homogeneous cones was founded by Koecher [2], Rothaus [3], Vinberg [4] and Gindikin [5].

Homogeneous cones that are also self-dual are called symmetric cones. They were studied much more than the general homogenous cones; their theory is presented in a book by Faraut and Koranyi [6].

In this paper we shall try to see how many of the results that hold for symmetric cones can be transferred to general homogeneous cones (and the latter class is considerably larger). This is based on Vinberg's theory of homogeneous cones, in which the elements of the cone are given in the form of "generalized matrices"; then everything becomes similar not only to symmetric cones, but to classical cones whose elements are positive definite matrices.

Vinberg's study is based on some nonassociative algebras, which are now called Vinberg algebras. The corresponding algebras for the symmetric cones are the Jordan algebras (see, for example, Faraut and Koranyi [6]). The symmetric cones were fully classified: there are 5 types of them. There are infinitely many types of general homogeneous cones, but their exact classification is not yet known. 
In sections 2 and 3 we review some properties of the homogeneous cones following mostly Vinberg [4]. As an example of an application of this theory to analysis we study integral transforms and their asymptotic behaviour. Analysis on homogeneous cones was developed, and to a much larger extent, by Gindikin [5]. We shall eventually reprove some of his results in the framework of Vinberg's theory. In this form these results look more similar to the ones for classical cones. In section 4 we consider the power functions which are the $n$-dimensional functions $t \mapsto t \alpha$; they also give the invariant measure and take part in the definition of homogeneous kernels. In sections 5 and 6 we deal with a class of integral transforms with homogeneous kernels and prove a theorem of Abelian type. Examples of these integral operators are the Laplace transform, the Riemann-Liouville operator and the Stieltjes transform.

\section{THE CONE, THE GROUP, THE LIE ALGEBRA AND THE VINBERG ALGEBRA}

In this section we review some notions we shall need from the theory of homogeneous cones. In particular, we shall see how the group of automorphisms of the cone and its Lie algebra determine a Vinberg algebra in the ambient space $\mathbb{R}^{n}$.

DEFINITION 2.1. Let $V$ be an open convex cone in $\mathbb{R}^{n}$, which doesn't contain any straight line. The cone $V$ is said to be homogeneous if there is a group $G$ of linear automorphisms (a subgroup of $G L(n, \mathbb{R})$ which leaves $V$ invariant) which is transitive on $V$; i.e., such that for every $u, v \in V$ there is an element $g$ of $G$ such that $v=g u$.

A most important property of homogeneous cones is that they always have a simply transitive group of automorphisms, i.e. a group $G$ such that for every $u, v \in V$ there is a unique $g \in G$ such that $v=g u$. In other words, there is a bijection $\Pi: G \rightarrow V$ which, if we fix an element $c \in V$ once for all, assigns to every $v \in V$ a unique $g_{v} \in G$ such that $v=g_{v} c$. Thus the group operation induces an operation for the elements of the cone

$$
v \cdot u=g_{v} g_{u} c \text {. }
$$

We shall call this simply transitive group $G$ the group of the cone. This group is triangular (real solvable) and if 9 is the Lie algebra of $G$, then the exponential mapping is a bijection from the Lie algebra 9 onto $G$. By taking the derivative of the mapping $\Pi$ above, we see that the mapping $\pi: g \rightarrow \mathbb{R}^{n}$ defined by $X \mapsto X c$ is a vector space isomorphism. Thus we have bijective mappings between the four sets: $V, G, 9$ and $\mathbb{R}^{n}$. In particular, if for $x \in \mathbb{R}^{n}$ we write $L(x)$ for the unique element of $g$ such that $x=L(x) c$, we can define the following operation

$$
x \Delta y=L(x) y=L(x) L(y) c .
$$

This bilinear operation introduces into $\mathbb{R}^{n}$ the structure of a Vinberg algebra. This is the nonassociative algebra described in the next definition.

Equation (2.2) gives an action of the Lie algebra 9 on $\mathbb{R}^{n}$. Also, by the bijectivity the exponential mapping, every $g \in G$ is of the form $g=\exp L(x)$, with some $L(x) \in 9$ and the corresponding action of the Lie group $G$ is given by

The orbit of the point $c$ is the cone $V$.

$$
g y=\exp L(x) y \text {. }
$$

DEFINITION 2.2. A Vinberg algebra $B=\left(\mathbb{R}^{n}, \Delta\right)$ is a vector space $\mathbb{R}^{n}$ with a bilinear operation $\Delta$ such that

(B) $\quad x \Delta(y \Delta z)-(x \Delta y) \Delta z=y \Delta(x \Delta z)-(y \Delta x) \Delta z$

(B.1) the operator of left multiplication $L(x): \beta \rightarrow ß \beta, L(x) y=x \Delta y$ has all the eigenvalues real,

(B.2) $\operatorname{Tr} L(x \Delta x)>0$, for every $x \in \beta$.

The Vinberg algebra has an identity $c$ if moreover

(B.3) $x \Delta c=c \Delta x=x$.

A Vinberg algebra $B$ with identity $c$ has a Pierce decomposition. First of all, there is a complete system of orthogonal idempotents $c_{1}, \ldots, c_{m}$, with $c_{1}+\ldots+c_{m}=c$; the number $m$ is called the rank of $\beta 3$. Then $B$ is decomposed into a direct sum of subspaces

$$
\boldsymbol{B}=\sum_{\mathrm{i} \leq \mathrm{j}} \boldsymbol{B}_{i j}, \quad i, j=1, \ldots, m
$$


where $\beta_{i i}=\mathbb{R} c_{i}$ and the subspace $\beta_{i j}$ is characterized by the fact that the operators $L\left(c_{i}\right)$ and $L\left(c_{j}\right)$ have the eigenvalue $1 / 2$ on it, and the operator $R\left(c_{j}\right)$ has the eigenvalue 1; all the other $L\left(c_{k}\right)$ and $R\left(c_{k}\right)$ are zero on $\beta_{i j}$. The subspaces $\beta_{i j}$ have dimensions $n_{i j}$.

We shall write $x_{i j}$ for an element of $\beta_{i j}$, and in particular, $x_{i i}=x_{i} c_{i}$, with $x_{i} \in \mathbb{R}$. Thus by (2.4), every $x \in \mathcal{B}$ is of the form

$$
x=\sum_{i \leq j} x_{i j}=\sum_{i} x_{i} c_{i}+\sum_{i<j} x_{i j}
$$

Then for $L(x)$ we have an analogous decomposition

$$
L(x)=\Sigma_{i} x_{i} L\left(c_{i}\right)+\Sigma_{i<j} L\left(x_{i j}\right) .
$$

Consider the Lie algebra 9 , which, as every triangular Lie algebra, is decomposed into the sum $9=$ $a+\eta$ of the abelian subalgebra $G$ and the nilpotent subalgebra $\Omega=[9,9]$. The operators $L\left(c_{i}\right)$ constitute a basis of the $\mathrm{G}$ - they commute since $c_{i}$ are orthogonal idempotents; this can be easily seen if we note that in terms of the operators of left multiplication condition (B) takes the form $[L(x), L(y)]=L\left(x_{\Delta} y-y_{\Delta} x\right)$. The elements $L\left(x_{i j}\right)$ belong to the nilpotent subalgebra $れ$. The trace of $L(x)$ equals

$$
\operatorname{Tr} L(x)=\sum_{i=1}^{\mathrm{m}} x_{i} \operatorname{Tr} L\left(c_{i}\right)=\sum_{\mathrm{i}=1}^{\mathrm{m}} x_{i} d_{i}
$$

where we have put $d_{i}=\operatorname{Tr} L\left(c_{i}\right)$ (since $\operatorname{Tr} L\left(x_{i j}\right)=0$ ). By the remark above about the eigenvalues of $L\left(c_{i}\right)$ it follows that

$$
d_{i}=1+v_{i} f 2+\mu_{i} / 2 \quad \text { with } \quad v_{i}=\sum_{\alpha<i} n_{\alpha i}, \quad \mu_{i}=\sum_{\beta>i} n_{i \beta}
$$

for $i=1, \ldots, m$. We shall write $d=\left(d_{1}, \ldots, d_{m}\right), v=\left(v_{1}, \ldots, v_{m}\right)$ and $\mu=\left(\mu_{1}, \ldots, \mu_{m}\right)$ for these three multi-indices, which are characteristic for the cone. We shall need them in sections 5,7 and 8 and the values of these indices for different examples of cones are given in section 9.

In a Vinberg algebra we can introduce an inner product by

$$
(x \mid y)=\operatorname{Tr} L(x \Delta y) \text {. }
$$

Condition (B2) shows that this bilinear form is positive definite. In particular, by (2.7) we see that for the identity $c$ we have

$$
(c \mid x)=\sum_{i=1}^{m} x_{i} d_{i}
$$

\section{THE MATRIX CALCULUS}

In this section we write an element $x$ of a Vinberg algebra, given as in (2.5), in the form of a symmetric "matrix" in the following way. We write the real numbers $x_{i}$ as diagonal entries and the $n_{i j}$ dimensional elements $x_{i j} \in \beta_{i j}$ in the $i j$ and $j i$ position. We thus define a mapping $m: \beta \rightarrow \Re$ from $\beta$ into the space of generalized symmetric matrices $\mathscr{H}$. The matrix product for two such matrices is defined by making use of the operation (2.2) (see Vinberg [4] for details). It is a nonassociative operation.

Let $\mathcal{J}$ be the subspace of upper triangular matrices in $\mathcal{H}$. If $A \in \mathcal{H}$, let $A \in \mathcal{J}$ be the "upper trangular half" of $A$, i.e. a matrix $\hat{A}$ such that $\mathrm{A}=\hat{A}+\hat{A}^{\top}$, where ${ }^{\top}$ is the transposition in this matrix algebra. For $A$, $B \in \mathcal{H}$ put

$$
A \Delta B=\hat{A} B+B \hat{A}^{\top} \text {. }
$$

THEOREM 3.1. (Vinberg [4]). The space $\mathscr{F}$ with the operation (3.1) is a Vinberg algebra and the mapping $m: B \rightarrow \mathcal{H}$ is an isomorphism of Vinberg algebras, i.e. $m(x \Delta y)=m(x) \Delta m(y)$.

Isomorphic Vinberg algebras have equal inner products (2.9). Thus for the algebras $\beta$ and $\mathscr{H}$ we have $(x \mid y)=(m(x) \mid m(y))$ and in particular, since $m(c)=I$, the identity matrix, we deduce from (2.10)

$$
(I \mid A)=\sum_{i=1}^{m} a_{i i} d_{i}
$$

where $A=m(x)$ and $a_{i i}\left(=x_{i}\right)$ are the diagonal elements of the matrix $A$

Now, consider the triangular subalgebra $\mathcal{\tau}$. It turns out that the restriction of the matrix product to $\mathcal{T}$ is associative. Then the commutator $[X, Y]=X Y-Y X$ makes $\gamma$ into a Lie algebra, which is isomorphic with 9. This isomorphism is given by the mapping $\psi: L(x) \mapsto X$ which writes the element $L(x)$ from $(2.6)$ in the form of an upper triangular matrix by putting $1 / 2 x_{i}$ in the diagonal position and $x_{i j}$ in the $i j$ position, for $i<j$. 
(The factor $1 / 2$ is introduced into the diagonal entries so that for $A=m(x)$ and $X=\psi(L(x))$ we have $A=X+$ $X^{\top}$.) Formula (3.1) actually defines the action of the Lie algebra $\mathcal{T}$ on $\mathscr{F}$ : if $X \in \mathcal{F}$ and $B \in \mathcal{F}$ the mapping

$$
p(X): B \mapsto X B+B X^{\top}
$$

acts on $\mathcal{F}$ and by Theorem 3.1 it is isomorphic with the action of the element $L(x)$ of the Lie algebra 9 defined in (2.2).

Now let $T=\exp \mathcal{J}$ be the Lie group with Lie algebra $\mathcal{J}$. It is easily seen, by standard arguments, that the exponential mapping is given by $\exp X=I+X+X^{2} / 2 !+X^{3} / 3 !+\ldots$, where $X^{2}=X X$, etc. is the matrix product. Then it follows that $T$ is the subset of $\mathcal{T}$ consisting of matrices with positive diagonal elements.

The groups $T$ and $G$ are isomorphic and the isomorphism $\Psi$ is the exponential of $\psi$, i.e.

$$
\Psi: g=\exp L(x) \mapsto t=\exp \psi(L(x)) .
$$

The exponential of the action $p(X)$ of (3.3) gives the following representation $P$ of $T$ in

$$
P(t): B \mapsto t\left(B t^{\top}\right)
$$

which is isomorphic to the action of $g=\exp L(x)$ in (2.3).

LEMMA 3.1. Let $t \in T$ be the image by $\Psi$ of $g=\exp L(x)$. Then the diagonal elements of $t$ are equal to $t_{i i}=\mathrm{e}^{x / 2}$.

Indeed, we are looking for the $i$-th diagonal element of $t=\exp \psi(L(x))$. Since $t$ is a triangular matrix, which is equal to the exponential of a triangular matrix $X=\psi(L(x))$, we have that $t_{i i}$ is equal to the exponential of the $i$-th diagonal element of $X$, which is equal to $x_{i} / 2$.

The orbit by $T$ of the point $I=m(c)$ is a cone $W$, isomorphic to $V$. Indeed, it is readilly seen that $W=$ $m(V)$. Thus every $w \in W$ is in $P(T) I$, in other words, there is a $t \in T$ such that

$$
w=t t^{\top} \text {. }
$$

Equation (3.6) is called the Gauss decomposition of the symmetric matrix $w$. Matrices having a Gauss decomposition will be also called "positive definite" (because the diagonal elements of $t$ are positive). Thus $W$ is the cone of positive definite matrices in $\mathscr{H}$. This is analogous to the case of the symmetric cones consisting of positive definite elements of the corresponding Jordan algebra. Note how here the Gauss decomposition $t t^{\top}$ is obtained immediately, whereas in Jordan algebras it requires some work (see Faraut and Koranyi [6]).

Let us close this section with a formula in which an instance of the matrix product is computed and which we shall need in section 7. For an upper triangular matrix $t$ whose matrix components are $t_{i j}$ the diagonal components of the product $t t^{\top}$ are equal to

$$
\left(t t^{\top}\right) i i=t_{i i}^{2}+\frac{1}{2 d_{i}} \sum_{j=i+1}^{m}\left|t_{i j}^{2}\right|
$$

where $\left\|t_{i j}\right\|^{2}=\left(t_{i j} \mid t_{i j}\right)$ (see Vinberg [4]). And now by (3.2) we have

$$
\left(I \mid t t^{\top}\right)=\sum_{i=1}^{m} t_{i i}^{2}+\frac{1}{2} \sum_{i<j}\left\|t_{i j}^{2}\right\| .
$$

\section{THE POWER FUNCTIONS}

The power functions are generalizations of the function $\tau \mapsto \tau^{\alpha}$ in one variable. They are in fact the group characters. For the classical cones the power functions are the principal minors of the matrix. This is generalized to any homogeneous cone, the main tool being the Gauss decomposition; if in $w=t t^{\top}$ we take the entries of the matrix $t$ as coordinates for $w$, then the power function has a particularly simple form - it is a product of diagonal coordinates (see (4.5) below).

Note first that the mapping $\Pi: G \rightarrow V$ gives a bijection between the functions $F: V \rightarrow \mathbb{R}_{+}$and $f: G \rightarrow$ $\mathbb{R}_{+}$in the following way

$$
F(v)=f\left(g_{v}\right) \quad \text { or } \quad f(g)=F(g c) .
$$

DEFINITION 4.1. A function $\Phi: V \rightarrow \mathbb{R}_{+}$is called a power function if the function $\phi: G \rightarrow \mathbb{R}_{+}$ defined in (4.1) is a group character.

It follows that

$$
\Phi(g v)=\phi(g) \Phi(v) \quad \text { and } \quad \Phi\left(v \cdot v^{\prime}\right)=\Phi(v) \Phi\left(v^{\prime}\right)
$$


for every $g \in G$ and $v, v^{\prime} \in V$ (where . is defined as in (2.1)). It is easy to determine the exact form of all power functions.

LEMMA 4.1. Let $\phi$ be a power function and let $g \in G$ be written as $g=\exp L(x)$, with $L(x)$ as in (2.6). Then there exist real numbers $\alpha_{i}$ such that

$$
\phi(g)=\mathrm{e}^{\alpha_{1} x_{1}+\ldots+\alpha_{m} x_{m}}
$$

If $\alpha=\left(\alpha_{1}, \ldots, \alpha_{m}\right)$ is a multi-index, we shall write $\phi^{\alpha}$ for the power function in (4.3) and $\Phi^{\alpha}$ for the power function related to $\phi^{\alpha}$ as in (4.2). Denote by $\phi_{i}$ and $\Phi_{i}$ the elementary power functions

Thus every $\phi^{\alpha}$ is equal to $\phi^{\alpha}=\phi_{1}^{\alpha_{1}} \ldots \phi_{m}^{\alpha_{m}}$.

$$
\phi_{i}(g)=\mathrm{e}^{x_{i}}, \quad \Phi_{i}(v)=\mathrm{e}^{x_{i}}
$$

Now let $T$ be the triangular matrix group from section 3, isomorphic with $G$. We shall write $\phi^{\alpha}$ for the power function on $T$ and $\Phi^{\alpha}$ for the corresponding power function on the cone $W=m(V)$. Then we have for $t=\Psi(g)$ as in (3.4)

$$
\begin{gathered}
\phi^{\alpha}(g)=\phi^{\alpha}(t)=t_{11}^{2 \alpha_{1}} \ldots t_{m m}^{2 \alpha} \\
\Phi^{\alpha}(v)=\Phi^{\alpha}(m(v))
\end{gathered}
$$

Indeed, it is obvious that isomorphic groups have equal characters. Now by Lemma 3.1 we have $t_{i i}=\mathrm{e}^{x / 2}$, and the second equality in (4.5) follows by (4.3).

An important example of a power function is the norm $N: V \rightarrow \mathbb{R}_{+}$defined as

$$
N(g v)=\operatorname{Det} g N(v) \text {. }
$$

(see Koecher [2]). Since for $g=\exp L(x)$ we have Det $g=\mathrm{e}^{\operatorname{Tr} L(x)}=\mathrm{e}^{\Sigma d_{1} x_{i}}$ by (2.7), we see that

$$
\operatorname{Det} g=\phi^{d}(g), \quad N(v)=\Phi^{d}(v)
$$

with $d$ the multi-index defined in (2.8).

As another example, take the multi-index $\mathbb{1}=(1, \ldots, 1)$ and put

$$
\Delta(v)=\Phi^{\mathbb{1}}(v), \quad \delta(g)=\phi^{\mathbb{l}}(g)=\mathrm{e}^{x_{1}+\ldots+x_{m}}
$$

and call this function the delta function of the cone.

REMARK 4.1. By applying (4.5) we see that when $v$ is an ordinary matrix (written as $v=t t^{\top}$ ), then the delta function $\Delta(v)$ is the ordinary determinant of $v$. Indeed, $\Delta(v)=\phi^{\mathbb{1}}(t)=\left(t_{11} \ldots t_{\text {mm }}\right)^{2}$ according to (4.5) and this equals (det $t)^{2}=\operatorname{det} v$. When in (4.8) the multi-index $d$ is a constant $d_{i}=d$, and this is the case only for symmetric cones, we obtain a formula $N(v)=(\Delta(v))^{d}$, well-known in Jordan algebras (see Faraut and Koranyi [6]); in the theory of Jordan algebras $\Delta(v)$ equals det $v$, the determinant of an element in the Jordan algebra. We write capital letters when Det and $\operatorname{Tr}$ are the determinant and the trace of a linear operator (in (4.8) $g$ is identified with the linear operator, as in (2.3)).

\section{AN INTEGRAL FORMULA}

In section 6 we shall deal with integral transforms of functions defined on $V$. The formula in Theorem 5.1 below will be used to compute some integrals. The Gauss decomposition (3.6) provides a good change of variables in an integral containing a power function, since the Jacobian for this change of variables is also a power function. We shall apply this formula in section 7 to compute the gamma function of the cone. For the classical cones this formula can be found in Garding [7]. It was generalized to the symmetric cones in Faraut and Koranyi [6]. Gindikin [5] has evaluated the integral for the gamma function by a different parametrization.

First consider the invariant measure on $V$. Here a measure $d m$ is called invariant if it is invariant under the action of $G: d m(g v)=d m(v)$, for every $g \in G, v \in V$. Then by (4.8) we find easily the following lemma.

LEMMA 5.1. The invariant measure on $V$ is equal to $d m(v)=d v / N(v)$.

Here $N$ is the norm of $V$ and, for $v$ written as in (2.5), $d v=d v_{1} \ldots d v_{m} d v_{12} \ldots d v_{m-1 m}$ is the Lebesgue measure on $\mathbb{R}^{n}$, where $d v_{i j}$ is the $n_{i j}$-dimensional Lebesgue measure on the space $\mathbb{R}^{n_{i j}} \cong \mathbb{B}_{i j}$.

We shall take the cone in its $W$ form (and write again $\phi^{\alpha}$ in the place of $\phi^{\alpha}$ ). The change of variables in the following theorem is given by $\Pi_{1}: T \rightarrow W$ with

$$
w=\Pi_{1}(t)=t t^{\top} \text {. }
$$


Recall that the multi-indices $d, v$ and $\mu$ were defined in (2.8).

THEOREM 5.1. Let $F: W \rightarrow \mathbb{R}_{+}$be integrable with respect to the invariant measure $d m$. Then

$$
\int_{W} F(w) d m(w)=2^{m} \int_{T} F\left(t t^{\top}\right) \phi^{-\gamma}(t) d t
$$

where $\gamma$ is the multi-index $\gamma=1 / 2+\mu / 2$.

PROOF. By Lemma 5.1 the invariant measure is given by the power function $\Phi^{-d}(w)=\phi^{-d}(t)$. Thus

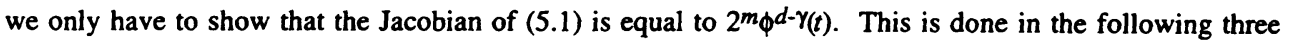
lemmas.

LEMMA 5.2. Let $l_{t}: T \rightarrow T$ be the left translation in the group: $l_{t}(s)=t s$ and let $\Pi_{1}$ be as in (5.1). Then

$$
\Pi_{1} \circ l_{t}=P(t) \circ \Pi_{1} .
$$

PROOF. Since $\Pi_{1}(t)=t t^{\top}=P(t) I$ (by (3.5)) we have for the left-hand side of (5.2) $\Pi_{1} \circ l_{(}(s)=$ $\Pi_{1}(t s)=P(t s) I$ and for the right-hand side of (5.2) we have $P(t) \circ \Pi_{1}(s)=P(t) P(s) I$. Since $P(t)$ is a group action, the lemma follows.

LEMMA 5.3. Let $\Pi_{1}(t)=t^{\top}$. Then $\operatorname{Det} d \Pi_{1}(t)=2^{m} \frac{\operatorname{Det} P(t)}{\operatorname{Det} l_{t}}$.

PROOF. Take the derivative of (5.2) at $I$

$$
d \Pi_{1}(t) \circ d l_{t}(I)=d P(t)(I) \circ d \Pi_{1}(I) .
$$

Since $P(t)$ and $l_{t}$ are linear transformations, they are equal to their derivatives. Now $d \Pi_{1}(I): \mathcal{J} \rightarrow \mathscr{H}$ is given by $X \mapsto X+X^{\top}$, and thus Det $d \Pi_{1}(I)=2^{m}$. By taking the deteminates of (5.3) the lemma follows.

LEMMA 5.4. If $l_{t}$ is the left translation in $T$, then Det $l_{t}=\phi^{\gamma}(t)$.

PROOF. Since $l_{t s}=l_{t} l_{s}$ we have that $\lambda(t)=\operatorname{Det} l_{t}$ is indeed a power function. Thus we only have to determine the exponent $\gamma$. By Lemma 4.1 a power function depends only on the abelian part. This means that if $a$ is the abelian part of $t \in T$, then $\lambda(t)=\lambda(a)$. Thus to compute $\lambda(a)$ consider $l_{a}: \mathrm{T} \rightarrow \mathrm{T}$. By Lemma 3.1 the elements of the diagonal matrix $a$ are $\mathrm{e}^{x / 2}$ so that $l_{a}$ multiplies the $i$-th row of $s \in T$ by a factor $\mathrm{e}^{x / 2}$. Then in the Jacobian we have this factor $\gamma_{i}$ times, where $\gamma_{i}$ is the sum of the dimensions in the $i$-th row, and this is equal to $1+\mu_{i}$ (see (2.8)).

Now to finish the proof of the theorem, we have to substitute in Lemma 5.3 the values of $\operatorname{Det}_{t}=\phi^{\gamma}(t)$ and $\operatorname{Det} P(t)$. Since $P(t)$ (3.5) is an action isomorphic with (2.3), we have $\operatorname{Det} P(t)=\operatorname{Det} g$ and this equals $\phi^{d}(g)=\phi^{d}(t)$. Finally the Jacobian is equal to $2^{m} \phi^{d-\gamma(t)}$. This proves the theorem.

\section{INTEGRAL TRANSFORMS WITH HOMOGENEOUS KERNELS}

Let $V \subseteq \mathbb{R}^{n}$ be a homogeneous cone and let $F: V \rightarrow \mathbb{R}_{+}$. We shall consider operators of the form

$$
\mathscr{K} F(v)=\int_{V} k(v, u) F(u) d m(u)
$$

where $k: V \times V \rightarrow \mathbb{R}+$ is a given function, the kernel of $\mathscr{K}$, and $d m$ is the invariant measure. The kernel is said to be homogeneous of order $\gamma$ if

$$
k(g v, g u)=\phi \gamma(g) k(v, u)
$$

for every $g \in G$ and $v, u \in V$, where $\phi \gamma$ is the power function. It is easily seen that if $\mathcal{K}$ has a homogeneous kernel, it transforms power functions into power functions.

LEMMA 6.1. Let $\mathcal{K}$ be an operator with homogeneous kernel of order $\gamma$. If the integral

$$
\kappa_{\alpha}=\int_{V} k(c, u) \Phi^{\alpha}(u) d m(u)
$$

is convergent, then the integral defining $\mathscr{K} \Phi \propto(v)$ is convergent for every $v \in V$ and

$$
\mathscr{K} \Phi \alpha(v)=\kappa_{\alpha} \Phi \alpha+\gamma(v) \text {. }
$$

PROOF. Equation (6.2) can be written as $k(v, u)=\Phi \gamma(v) k\left(c, g_{v}{ }^{-1} u\right)$. Now by putting this into the definition of $\mathscr{K F}(v)$ and changing the variable $y=g_{v}{ }^{-1} u$ we obtain

$$
\mathscr{K F}(v)=\Phi(v) \int_{V} k(c, y) F(v \cdot y) d m(y) .
$$

Finally put $F=\Phi^{\alpha}$ in this formula and use (4.2)

Postponing until the next section the computation of $\kappa_{\alpha}$ for some examples of operators (6.1), we are now going to prove that if a function $F$ is asymptotically equivalent to a power function, then its integral 
transform $\mathscr{K F}$ is asymptotically equivalent to a power function. To define limits we consider translation invariant filters.

DEFINITION 6.1. A filter $\mathscr{S}$ of open convex subsets of $V$ is called translation invariant if it has a countable basis and if, for every $y \in V$, we have that $S \in \mathscr{S}$ implies $S . y \in \mathscr{S}$.

We shall write $v \rightarrow \infty$ for the convergence with respect to this filter. Thus we have that $v \rightarrow \infty$ implies $v \cdot y \rightarrow \infty$, for every $y \in V$.

Two functions $F, G: V \rightarrow \mathbb{R}_{+}$are said to be equivalent at infinity with respect to $\mathscr{S}$, if $\lim _{v \rightarrow \infty} F(v) / G(v)$ $=1$. This will be denoted by $F \sim G$.

REMARK 6.1. A different kind of asymptotic relations for functions defined on cones in $\mathbb{R}^{n}$ were considered in Vladimirov et al. [8] and Yakymiv [9]. The notion of limit in Definition 6.1 is more general (it is taken after Bajsanski and Karamata [10]). Indeed, by the identification (4.1), it is equivalent to consider a filter of subsets in $G$ and then, for example, $\lim _{v \rightarrow \infty} F(v \cdot y)$ is the same as $\lim _{g \rightarrow \infty} F(g y)$. In our notation, the convergence in Vladimirov et al. [8] becomes convergence with respect to one parameter subgroups $g(t)$ (with $t \rightarrow \infty$ ) in $G$. The asymptotic in Yakymiv [9] is even more special, with $g(t)$ the group of dilations. (Compare also with Stadtmüller [11], where $g(t)$ is an arbitrary curve, but only in the cone $\mathbb{R}_{+}^{n}$.) Both in Vladimirov et al. [8] and Yakymiv [9] the cone is only supposed to be (the closure of) an open, convex cone, and it has less structure than a homogeneous cone.

THEOREM 6.1. Let $\mathscr{K}$ be an integral operator with homogeneous kernel of order $\gamma$. Let $F: V \rightarrow$ $\mathbb{R}_{+}$be such that $F \Phi^{-\alpha}$ is bounded. Then

$$
F \sim \Phi \alpha \quad \text { implies } \quad \mathscr{K} F \sim \kappa_{\alpha} \Phi^{\alpha+\gamma}
$$

for those $\alpha$ for which the integral $k_{\alpha}=\mathscr{K} \Phi \alpha(c)$ is convergent.

We shall first consider the case when $\alpha=0$. Then $F(v) \rightarrow 1, v \rightarrow \infty$. Since we are only interested in the asymptotic behaviour, we may modify the functions so that they become bounded on the complements of the elements of the filter basis (without changing its behaviour at $\infty$ ). Together with $F(v) \rightarrow 1$, this modification yields that $F$ is bounded everywhere.

PROPOSITION 6.1. Let $\mathscr{K}$ be an integral operator with homogeneous kernel of order $\beta$ and such that $\kappa_{0}<\infty$. Let $F: V \rightarrow \mathbb{R}_{+}$be bounded. Then $F(v) \rightarrow 1$, when $v \rightarrow \infty$ implies

$$
\mathscr{R F} \sim \kappa_{0} \Phi \beta \text {. }
$$

(Here $\kappa_{0}$ is defined in (6.3), with $\Phi^{0}=1$.)

PROOF. By applying (6.5) we have that (6.6) is equivalent to the fact that the following expression tends to zero

$$
\Phi-\beta(v) \mathscr{K} F(v)-\kappa_{0}=\int_{V} k(c, y)(F(v \cdot y)-1) d m(y) .
$$

Now for $\varepsilon>0$ take a (large) compact set $D \subseteq V$ such that

$$
\int_{V-D} k(c, y) d m(y)<\varepsilon
$$

which is possible by the assumption $\kappa_{0}<\infty$, and write the integral in (6.7) as

$$
\int_{V}=\int_{D}+\int_{V-D}=I_{1}+I_{2}
$$

To estimate $I_{1}$, note that, by the translation invariance of the filter, $v \rightarrow \infty$ implies $v \cdot y \rightarrow \infty$, for every $y$, so that $F(v \cdot y)$ tends to 1 , when $v \rightarrow \infty$, and in fact uniformly in $y \in D$. Thus we can find an $S \in \mathscr{S}$ so that for $v \in S$ we have

$$
\left|I_{1}\right|<\varepsilon \int_{D} k(c, y) d m(y) \leq \varepsilon \int_{V} k(c, y) d m(y)=\varepsilon \kappa_{0} .
$$

For $I_{2}$, since $F$ is bounded (by $C$, say), we have

$$
\left|I_{2}\right|<(C+1) \int_{V-D} k(c, y) d m(y)<\varepsilon(C+1)
$$

by (6.8). This proves the proposition.

PROOF OF THEOREM 6.1. Put $F_{1}(y)=F(y) \Phi^{-\alpha}(y)$ and $k_{1}(c, y)=\Phi^{\alpha}(y) k(c, y)$. Then we have $\mathscr{K}_{1} F_{1}(v)=\mathscr{K} F(v)$ and we are going to apply Proposition 6.1 to the operator $\mathscr{K}_{1}$. By the assumptions of the theorem, $F_{1} \rightarrow 1$ and is bounded, so that it satisfies the conditions of the proposition. The kernel $k_{1}$ is homogeneous of order $\beta=\alpha+\gamma$ and $\mathscr{K}_{1}$ satisfies the conditions of the proposition, since the constant $\kappa_{0}$ for 
this operator equals $\mathscr{K}_{1} \Phi^{0}(c)=\mathscr{K}^{\alpha}(c)=\kappa_{\alpha}$ and is finite. Now an application of the propostion gives $\mathscr{K}_{1} F_{1} \sim \mathcal{K}_{\alpha} \Phi^{\beta}$ and since $\beta=\alpha+\gamma$, we finally find $\mathscr{K} F=\mathscr{K}_{1} F_{1} \sim \mathcal{K}_{\alpha} \Phi^{\alpha+\gamma}$, which proves the theorem.

\section{THE LAPLACE TRANSFORM}

In this and the following two sections we consider some examples of the operators (6.1). They are $n$ dimensional analogues of some classical operators and were considered by Gindikin [5]. Here we shall apply Vinberg's theory of homogeneous cones to compute the integrals as in (6.3).

To define the Laplace transform, consider first the norm $N$ like in (4.7) and the inner product defined in (2.9), and for $v \in V$ let $v^{*} \in \mathbb{R}^{n}$ be such that for every $y \in \mathbb{R}^{n}$ we have $\left(v^{*} \mid y\right)=d \log N(v) y$. (See Koecher [2] or Rothaus [3]; actually $v^{*}$ belongs to the dual cone of $V$.) The mapping ${ }^{*}$ has the property

for every $g \in G$ and $v, u \in V$.

$$
((g v) * \mid g u)=(v * \mid u)
$$

The Laplace transform is defined by

$$
\mathscr{L} F(v)=\int_{V} \mathrm{e}^{-\left(v^{*} \mid u\right)} F(u) d m(u) .
$$

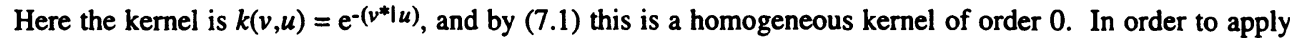
Theorem 6.1 to this operator we need to know the integral

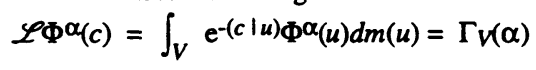

which is called the "gamma function of the cone" (see Koecher [2] or Gindikin [5]).

PROPOSITION 7.1. Let $\alpha$ be a multi-index such that $\alpha>\mu / 2$. Then the integral defining $\Gamma_{V}(\alpha)$ is convergent.

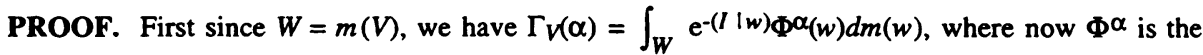
power function of $W$, which was denoted before by $\Phi^{\alpha}$ (see (4.6)), dm is the invariant measure on $W$ and the inner products on two isomorphic cones are equal. Now in this integral we put $w=t t^{\top}(\operatorname{see}(5.1))$. By Theorem 5.1 we have

$$
\Gamma_{V}(\alpha)=2^{m} \int_{T} \mathrm{e}^{-\left(l \mid t t^{\top}\right)} \phi^{\alpha}(t) \phi^{-\gamma(t) d t}
$$

Now we substitute (3.7) and (4.5) into the integral (7.2) and put $\delta_{i}=\alpha_{i}-\gamma_{i}$. Then we have

$$
\Gamma_{K}(\alpha)=\int \mathrm{e}^{-\Sigma d_{i} t_{i u}^{2}-1 / 2 \Sigma_{i<j}\left|t_{i j}\right|^{2}} t_{11}^{2 \delta_{1}} \ldots t_{m m}^{2 \delta_{m}} d t
$$

This integral is a product of one-dimensional integrals of two types. The first type

$$
I_{i}=\int_{0}^{\infty} \mathrm{e}^{-\Sigma d_{i} t_{i i}{ }^{2}} t_{i i}^{2 \delta_{1}} d t_{i i}=\frac{1}{2 d_{i}^{\delta_{i}+1 / 2}} \int_{0}^{\infty} \mathrm{e}^{-\tau} \tau^{\delta}{ }^{-1 / 2} d \tau
$$

is convergent since $\delta_{i}+1 / 2=\alpha_{i}-\gamma_{i}+1 / 2=\alpha_{i}-1 / 2-\mu_{i} / 2+1 / 2=\alpha_{i}-\mu_{i} / 2>0$, by the assumption.

The second type of integrals in (7.3) is

$$
I_{i j}=\int_{-\infty}^{\infty} \mathrm{e}^{-1 / 2\left(t_{i j}^{k}\right)^{2}} d t_{i j}^{k}
$$

where $t_{i j}^{k}$ are the coordinates of $t_{i j}$ in some basis in $\beta_{i j}$. By a well-known formula, $I_{i j}=(2 \pi)^{1 / 2}$. This proves the proposition.

Now an application of Theorem 6.1 gives the following.

THEOREM 7.1. (Abelian Theorem for the Laplace Transform). Let $\alpha>\mu / 2$. Let $F: V \rightarrow \mathbb{R}_{+}$be such that $F \Phi-\alpha$ is bounded. Then

$$
F \sim \Phi^{\alpha} \quad \text { implies } \quad \mathscr{L} F \sim \Gamma_{V}(\alpha) \Phi \alpha .
$$

\section{THE RIEMMAN-LIOUVILLE OPERATOR AND THE STIELTJES TRANSFORM}

In this section we define two more operators. Again we shall have to prove that the integrals (6.3) for these operators are convergent. But now there is no need to evaluate these integrals again, since they are generalizations of the beta function and can be obtained by reducing to the gamma functions, by formulas completely analogous to the classical ones. This was done in Gindikin [5]; for completeness we reproduce briefly these formulas. 
The cone $V$ defines a partial order in $\mathbb{R}^{n}$ in the following way: $v<u$ iff $u-v \in V$. Write $(a, b)$ for the "interval" with respect to this order, i.e. for the set of all elements $v \in V$ such that $a<v<b$.

Let $\beta$ be a multi-index, then the Riemann-Liouville operator is defined by

$$
\mathscr{S}_{\beta} F(v)=\int_{(0, v)} \Phi \beta(v-u) F(u) d m(u)
$$

where the kernel $k(v, u)=\chi_{(0, v)}(u) \Phi^{\beta}(v-u)$ is homogeneous of order $\beta$. Here $\chi$ is the characteristic function; and since $g \in G$ preserves the cone, it also preserves the order: $u<v$ implies $g u<g v$.

LEMMA 8.1. Let $\alpha>\mu / 2$ and $\gamma>\mu / 2$. Then the integral is convergent

$$
\int_{(0, c)} \Phi \alpha(u) \Phi \gamma-d(c-u) d m(u)=\mathrm{B}_{V}(\alpha, \gamma)
$$

This is easily seen if we consider the double integral defining $\Gamma_{V}(\alpha) \Gamma_{V}(\gamma)$, which by Proposition 7.1 is convergent for $\alpha>\mu / 2$ and $\gamma>\mu / 2$. By changing the order of integration this integral is equal to

$$
\Gamma(\alpha) \Gamma v(\gamma)=\int_{V} \mathrm{e}^{-(c \mid v)} \int_{(0, v)} \Phi \alpha(u) \Phi \gamma-d(v-u) d m(u) d v
$$

and by Fubini's theorem the inner integral is convergent for every $v \in V$ and for $\alpha$ and $\gamma$ as above.

THEOREM 8.1. (Abelian Theorem for the Riemann-Liouville Operator). Let $\beta>-1-v / 2$ and let for $\alpha>\mu / 2$ the function $F: V \rightarrow \mathbb{R}_{+}$be such that $F \Phi^{-\alpha}$ is bounded. Then

$$
F \sim \Phi \alpha \quad \text { implies } \quad \mathscr{4} \beta \sim B_{V}(\alpha, \beta+d) \Phi \alpha+\beta
$$

Let $\rho$ be a multi-index. Then the Stieltjes transform is defined by

$$
\mathscr{I}_{\rho} F(v)=\int_{V} \Phi^{-\rho}(v+u) F(u) d m(u)
$$

and its kernel $k(v, u)=\Phi-\rho(v+u)$ is homogeneous of order $-\rho$.

LEMMA 8.2. Let $\rho>\mu$ and $\mu / 2<\alpha<\rho-\mu / 2$. Then the following integral is convergent

$$
\int_{V} \Phi-\rho(c+u) \Phi \alpha(u) d m(u) \text {. }
$$

This is seen by introducing the change of variables $u=c+y$, and after that the change $x=u^{-1}=8 u^{-1} c$ (the Jacobian is equal to $\operatorname{Det}\left(g^{-2}\right)=\phi^{-2 d}(g)$, see Gindikin [5]). Then we obtain $B(p-\alpha, \alpha)$.

THEOREM 8.2. (Abelian Theorem for the Stieltjes Transform). Let $\rho>\mu$ and let for $\mu / 2<\alpha<\rho$ $\mu / 2$ and let the function $F: V \rightarrow \mathbb{R}_{+}$be such that $F \Phi^{-\alpha}$ is bounded. Then

$$
F \sim \Phi \alpha \quad \text { implies } \quad \mathscr{I}_{\rho} F \sim \mathrm{B}_{V}(\rho-\alpha, \alpha) \Phi \alpha-\rho
$$

\section{EXAMPLES OF CONES}

In this section we consider some examples of homogeneous cones. By Vinberg's matrix calculus we find the explicit form of the power function in much the same way as for the cones of ordinary matrices in example 1) below. We also write down the exact values of the constants $\mu_{i}, v_{i}$ and $d_{i}$ appearing in the formulation of the Abelian Theorems.

1) The Classical Cones. Let $\mathbb{R}^{n}=\mathscr{G}_{m}(F)$ be the space of symmetric $m \times m$ matrices with coefficients in $F=$ $\mathbb{R}, \mathbb{C}$ or $\mathbb{H}$. Let $V$ be the cone of positive definite matrices $\boldsymbol{P}_{m}(\mathbf{F})$. Here $x \in \mathbb{R}^{n}$ is already given in its matrix form. We have $n_{i j}=1,2$ or 4 , for $F=\mathbb{R}, \mathbb{C}$ or $\mathbb{H}$, respectively, and if we put $b=1,2$ or 4 , then $v_{i}=b(i-1)$ and $\mu^{i}=b(m-\mathbf{i})$. Then $d_{i}=d$ is constant, and equals $(m+1) / 2$ in $\mathbb{R}$; equals $m$ in $\mathbb{C}$; and equals $2 m-1$ in $\mathbb{H}$.

If $v \in V$, then $v=t t^{\top}$ is the well-known representation of a positive definite matrix. We know by (4.5) that $\Phi_{i}(v)=t_{i i}{ }^{2}$. Then the delta function equals $\Delta(v)=t_{11}{ }^{2} \ldots t_{m m}{ }^{2}$; i.e. it is equal to the determinant of $v$ and the norm is $N(v)=\Phi_{1}{ }^{d}(v) \ldots \Phi_{m}{ }^{d}(v)=(\Delta(v))^{d}=(\operatorname{det} v)^{d}$.

2) The Light Cone. This is the only symmetric cone not having a classical representation as a matrix cone. It is the set $V \subseteq \mathbb{R}^{n}$ of all elements $v$ such that $v_{1} v_{2}-v_{3}^{2}-\ldots-v_{n}^{2}>0$ and $v_{2}>0$. Here $m=2$. The matrices from section 3 are $2 \times 2$ matrices $A$ with $a_{11}=v_{1}, a_{22}=v_{2}$ and $a_{12}=\left(v_{3}, \ldots, v_{n}\right)$. Thus we have $n_{12}=n-2$ and $n_{1}=0, n_{2}=n-2, n^{1}=n-2$ and $n^{2}=0$. It follows that $d_{1}=d_{2}=n / 2$.

Now $A=t t^{\top}$ with $t$ an upper triangular matrix of the same type. In this product the entries of the form $t_{i i} t_{12}$ are scalar by vector products and $t_{12} t_{12}$ is the inner product of two (n-2)-vectors. If we write down this product we find

$$
\Phi_{1}(v)=t_{11}^{2}=a_{11}-a_{12} a_{12} / a_{22}=v_{1}-\left(v_{3}^{2}+\ldots+v_{\mathrm{n}}^{2}\right) / v_{2} \quad \Phi_{2}(v)=t_{22}{ }^{2}=a_{22}=v_{2} \text {. }
$$

The delta function is $\Delta(v)=\Phi_{1}(v) \Phi_{2}(v)=v_{1} v_{2}-v_{3}^{2}-\ldots-v_{n}^{2}$, and the norm $N(v)=(\Delta(v))^{n / 2}$. 
3) The Non-selfdual Cone in $\mathbb{R}^{5}$. This is the first non-selfdual cone (of smallest possible dimension). It is the set $V$ of all elements $v \in \mathbb{R}^{5}$ such that $v_{1} v_{3}-v_{4}^{2}>0, v_{2} v_{3}-v_{5}^{2}>0$ and $v_{3}>0$. In fact these inequalities are found by starting with the "matrices", which are now of the form

$$
\left[\begin{array}{ccc}
a_{11} & 0 & a_{13} \\
0 & a_{22} & a_{23} \\
a_{13} & a_{23} & a_{33}
\end{array}\right]
$$

with all entries real. Here we have $n_{12}=0$ and $n_{13}=1=n_{23}$ (it is indeed the smallest possible choice with $n_{i j}$ different). Thus we have $d_{1}=d_{2}=3 / 2$ and $d_{1}=2$. Such matrices are multiplied as ordinary matrices, except that we always write 0 in the 12 position: for example $a_{13} b_{32}=0$ and $a_{23} b_{31}=0$. A matrix $A$ is an element of the cone if it is repesentable as $A=t t^{\top}$ with $t$ an upper triangular matrix of the same type. Then we compute the elementary power functions

$$
\Phi_{1}(v)=t_{11}{ }^{2}=a_{11}-a_{13}{ }^{2} / a_{33} \quad \Phi_{2}(v)=t_{22}{ }^{2}=a_{22}-a_{23}{ }^{2 / a_{33}} \quad \Phi_{3}(v)=t_{33}{ }^{2}=a_{33} .
$$

Write $\left(v_{1}, v_{2}, v_{3}, v_{4}, v_{5}\right)=\left(a_{11}, a_{22}, a_{33}, a_{12}, a_{13}\right)$; then the conditions $\Phi_{i}(v)>0$ give the defining inequalities of the cone. The norm is equal to $N(v)=\Phi_{1}(v)^{3 / 2} \Phi_{2}(v)^{3 / 2} \Phi_{3}(v)^{2}$.

ACKNOWLEDGEMENT. I acknowledge gratefully the support of the Science Fund of Serbia through Grant 0401A.

\section{REFERENCES}

[1] WIDDER, D.V. The Laplace Transform, Princeton Univ. Press, Princeton, 1946.

[2] KOECHER, M. Posivitätsbereiche im $\mathbb{R}^{\text {n }}$, Amer. J. Math. 79 (1957), 575-596.

[3] ROTHAUS, O. Domains of Positivity, Abh. Math. Sem. Univ. Hamburg, 24 (1960), 189-235.

[4] VINBERG, E.B. The Theory of Homogeneous Convex Cones, Trudy Moskov. Mat. Obshch. 12 (1963), 303-358 (Trans. Moscow Math. Soc. 12 (1965)).

[5] GINDKIN, S.G. Analysis in Homogeneous Domains, Uspekhi Mat. Nauk XIX, No 4 (1964), 3-92 (Russian Math. Surveys 19 (1964), $\mathrm{N}^{\circ} 4$ )

[6] FARAUT, J. and KORANYI, A. Analysis on Symmetric Cones, Oxford University Press, Oxford, 1994.

[7] GARDING, L. The Solution of Cauchy's Problem for Two Totally Hyperbolic Linear Differential Equations by Means of Riesz Integrals, Ann. Math. 48 (1947), 785-826.

[8] VLADIMIROV, V.S., Drozzinov, Y.N. and Zavjalov, B.I. Multidimensional Tauberian Theorems for Generalized Functions, Nauka, Moscow, 1986.

[9] YAKYMIV, A.L. Multidimensional Tauberian Theorems and their Application to the Belman-Harris Branching Processes, Mat. Sb. 115 (157), N N $^{\circ}$ (7) (1981), $463-477$ (Russian Acad. Sci. Sb. Math. 115 (1981), $\left.N^{\circ} 3\right)$.

[10] BAJSANSKI, B. and KARAMATA, J. Regularly Varying Functions and the Principle of Equicontinuity, Publ. Ramanujan Inst. 1 (1969), 235-246.

[11] STADTMULLER, U. A Refined Tauberian Theorem for Laplace Transform in Dimension $d>1$, J. reine u. angew. Math. 328 (1981), 72-83. 


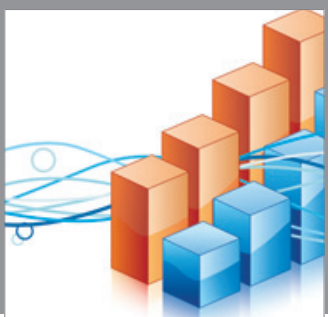

Advances in

Operations Research

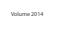

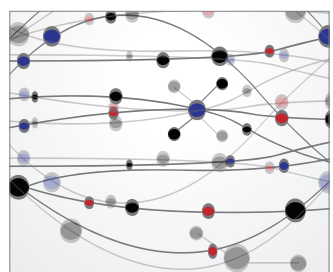

\section{The Scientific} World Journal
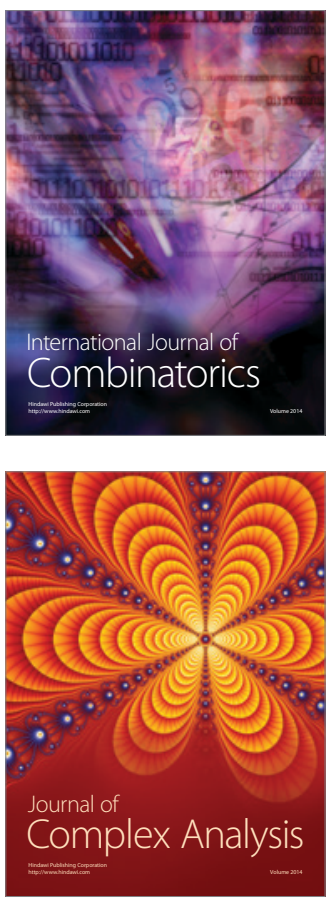

International Journal of

Mathematics and

Mathematical

Sciences
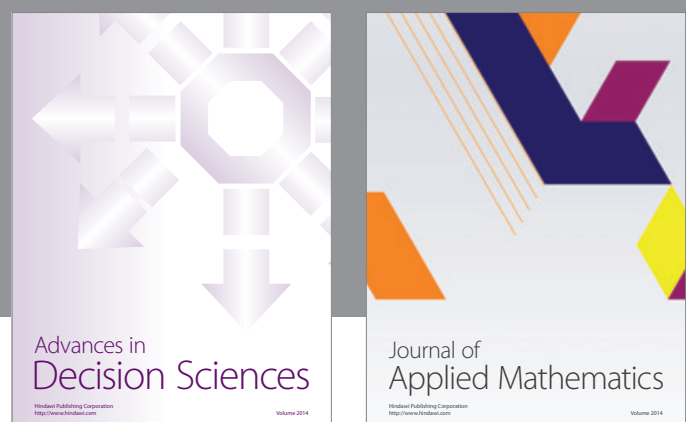

Journal of

Applied Mathematics
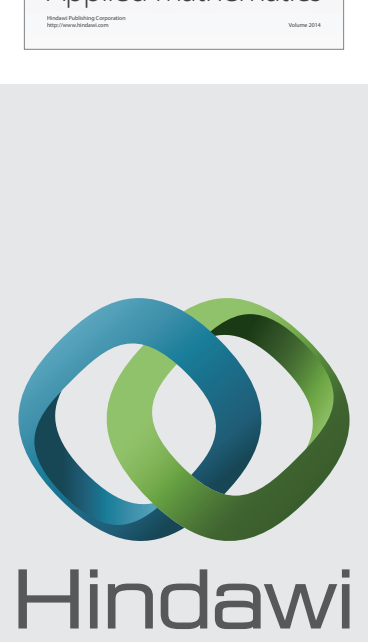

Submit your manuscripts at http://www.hindawi.com
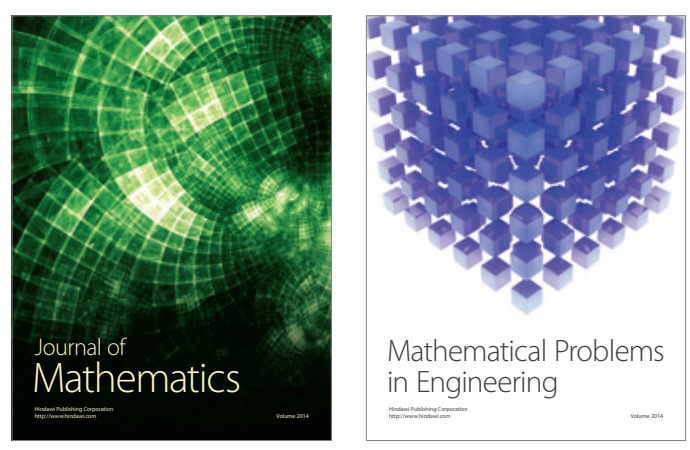

Mathematical Problems in Engineering
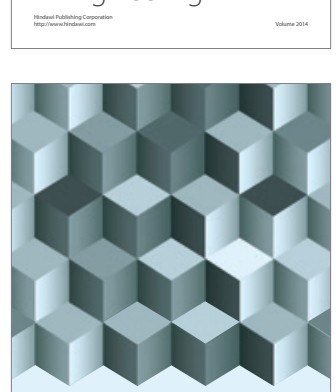

Journal of

Function Spaces
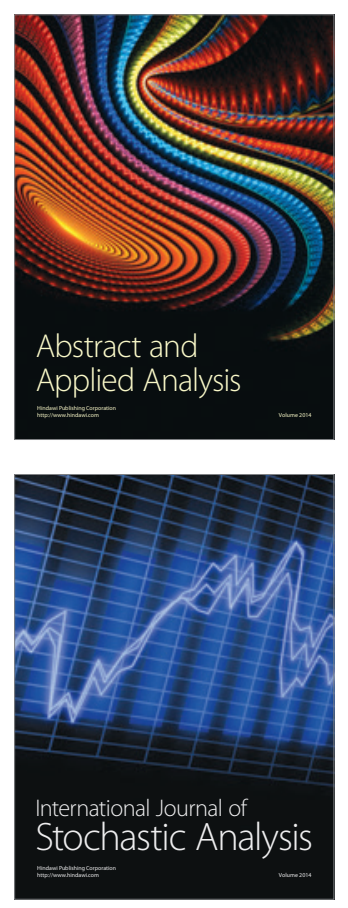

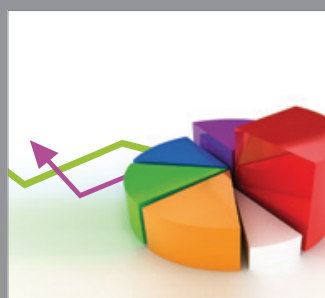

ournal of

Probability and Statistics

Promensencen
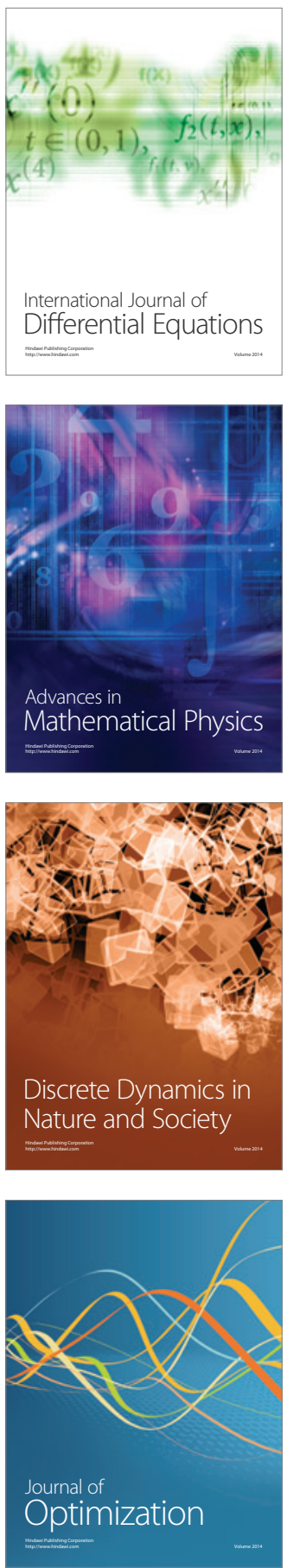